\title{
Use of negative pressure wound therapy as an adjunct to the treatment of extremity soft-tissue sarcoma with ulceration or impending ulceration
}

\author{
YU CHEN, SONG-FENG XU, MING XU and XIU-CHUN YU \\ Department of Orthopedic Surgery, General Hospital of Jinan Military Commanding Region, \\ Jinan, Shandong 250031, P.R. China
}

Received March 18, 2015; Accepted April 29, 2016

DOI: $10.3892 / \mathrm{ol} .2016 .4654$

\begin{abstract}
Major wound complications of the extremities, following wide tumorresection and reconstruction for soft-tissue sarcomas (STSs), remain a challenge for limb-sparing surgery. Furthermore, STSs with ulceration or impending ulceration predispose patients to an increased risk of post-operative infection. The present study was conducted to assess the efficacy of negative pressure wound therapy (NPWT) in preventing wound complications associated with surgical treatment of STSs with ulceration or impending ulceration, in patients treated between February 2012 and January 2013. A total of 5 patients, with a mean age of 48 years (range, 24-68 years), were enrolled in the present study. The diagnoses consisted of undifferentiated pleomorphic sarcoma $(n=2)$, leiomyosarcoma $(n=1)$, synovial sarcoma $(n=1)$ and epithelioid sarcoma $(n=1)$. According to American Joint Committee on Cancer criteria, 3 cases were stage III tumors, and the remaining 2 cases were of stages IIA and IIB, respectively. A total of 3 patients exhibited ulceration at diagnosis, and the remaining patients demonstrated impending ulceration. The mean wound area following wide resection of the tumor was $73 \mathrm{~cm}^{2}$ (range, $45-110 \mathrm{~cm}^{2}$ ). A continuous suction mode, with pressures measuring -200 to $-300 \mathrm{mmHg}$, was used for 7-10 days on the soft-tissue defects as preparation for wound closure. Soft-tissue reconstruction included muscle flaps $(n=2)$ and skin grafts $(n=5)$. No major wound complications occurred. Post-operative functional and cosmetic outcomes were acceptable. A single patient demonstrated local recurrence 12 months after surgery and re-excision of the tumor was performed. All patients remained alive at the conclusion of follow-up, with a mean follow-up
\end{abstract}

Correspondence to: Professor Xiu-Chun Yu, Department of Orthopedic Surgery, General Hospital of Jinan Military Commanding Region, 25 Shifan Road, Jinan, Shandong 250031, P.R. China

E-mail: toeflmugou@163.com

Key words: soft-tissue sarcoma, surgery, negative pressure wound therapy, vacuum-assisted closure time of 26 months (range, 12-36 months). The present study demonstrated that NPWT is effective and safe when used as an adjunct to wound closure following resection of extremity STS with ulceration/impending ulceration.

\section{Introduction}

Soft-tissue sarcomas (STSs) are a heterogeneous group of solid malignancies arising from the muscles, fat, connective tissue, nerves and blood vessels. The global incidence of STS is $\sim 5$ cases per 100,000 individuals $(1,2)$. The most common anatomical sites of occurrence are the extremities, followed by the trunk and retroperitoneum (3). En bloc resection of tumors along with a cuff of healthy tissue is the typical treatment for STS, as incomplete excision with positive microscopic surgical margins has been observed to be the strongest risk factor for local recurrence and distant metastasis (4). Due to an increased understanding of the pathology of STS, the evolution of surgical techniques and the development of adjuvant therapies, including radiotherapy (RT) and chemotherapy (CT), limb-sparing surgery has become the preferred choice of treatment due to an improved preservation of limb function and a post-operative prognosis equivalent to amputation (3-5). At present, only $10 \%$ of patients with STS of the extremities undergo amputation surgery (5).

However, when the tumor is in close proximity to the skin layer, with or without ulceration, bacterial colonization and inflammation may exist pre-operatively within the surgical field, which theoretically increases the risk of post-operative infection (4-6). Furthermore, wide excision of STS of the extremities results in a large tissue deficit that is typically unsuitable for primary closure; thus, soft-tissue reconstruction is necessary for limb-sparing surgery in these cases (6). This adds to the difficulty of surgical techniques, therefore, patients are expected to demonstrate an increased risk of major wound complications. Previous studies have reported a wide variety of wound complications following reconstruction, including cellulitis, abscess, wound dehiscence, seroma, hematoma and necrosis of the muscle flap (1-7). These complications can lead to unfavorable outcomes of treatment and postponed adjuvant therapy.

Therefore, proactive wound-care measures are imperative to decrease the risk of wound complications associated 
with reconstruction following wide resection of STSs with ulceration or impending ulceration. Negative pressure wound therapy (NPWT), also known as vacuum-assisted closure, is a revolutionary technique for the management of complex wounds. Previous studies have focused on the merits of NPWT compared with traditional methods in the treatment of complex wound defects (8). Despite a lack of high-level evidence, studies have indicated that NPWT may facilitate wound healing, prepare the wound bed for skin grafts or flaps, decrease the risk of infection and reduce the labor of clinicians (9-11). Conventional indications for NPWT include traumatic wounds, wounds with acute or chronic infections, ulcers, diabetic foot ulcers, dehiscent wounds and burns (12). However, to the best of our knowledge, there have been no reports concerning the application of NPWT as an adjunct to the treatment of extremity STS with skin involvement. In the present study, this novel wound care measure was used on tissue-deficient wounds caused by wide resection of extremity STS (with ulceration or impending ulceration) as preparation for secondary wound closure. The efficacy of NPWT in reducing major wound complications was assessed.

\section{Patients and methods}

Patient data. Between February 2012 and January 2013, 5 patients with extremity STS with skin involvement (ulceration or impending ulceration) were enrolled in the present study. The data of the patients is summarized in Table I. The cohort consisted of 4 men and 1 woman, with a mean age of 48 years (range, 24-68 years) and diagnoses of undifferentiated pleomorphic sarcoma $(n=2)$, leiomyosarcoma $(n=1)$, synovial sarcoma $(n=1)$ and epithelioid sarcoma $(n=1)$. The tumor sites were the forearm $(n=2)$, thigh $(n=1)$, knee $(n=1)$ and leg $(n=1)$. A total of 3 patients presented with ulcers on initial examination, and the remaining patients exhibited excessive skin tension, marked skin thinning, vanishing of subcutaneous fat and pigmentation, which indicated impending ulceration. At diagnosis, 3 patients presented with recurrent STS and previous surgery on the tumor sites. All STSs were localized tumors, and no metastases or comorbidities were detected. The mean duration of follow-up was 26 months (range, 12-36 months).

Pre-operative assessment. The pre-operative evaluation assessed a variety of factors, including overall health status, limb function, tumor location, size and depth, tumor involvement with major neurovascular structures, histological subtype of tumor and distant metastasis (typically lung metastasis). Prophylactic intravenous antibiotics [cephazolin, $1.0 \mathrm{~g}$ intravenously (IV), $30 \mathrm{~min}$ prior to surgery] were used in all cases, and a routine bacterial culture was performed pre-operatively in cases with ulceration as an indication for intravenous antibiotic use. Fine-needle aspiration biopsy was performed pre-operatively to determine the histological diagnosis. In accordance with the American Joint Committee on Cancer staging system (13), there were 3 stage III cases, 1 case of stage IIA and 1 case of stage IIB.

Surgery and NPWT procedure. Wide tumor resection with a radial 2 to $4-\mathrm{cm}$ margin was performed in all cases. Negative surgical margins were achieved according to the findings of the post-operative pathological examination. Immediate or secondary gastrocnemius muscle flap reconstruction was performed in 2 patients to cover the exposed bone and neurovascular structures. To reduce the risk of major wound complications and to prepare the wound bed for secondary soft-tissue reconstruction, a NPWT device (Smith \& Nephew Medical Ltd, Hull, UK) was applied as coverage on the wound deficit. Polyurethane ether foam $(15 \times 10 \times 1 \mathrm{~cm})$ was cut in accordance with the shape of the wound deficit and was used as a dressing. In the majority of cases, 1 piece of foam was sufficient to span the wound surface. The dressing was cut to cover the undetermined areas and tracks for dead cavity closure. All foam dressings were fixed to the skin edge by suturing. Continuous NPWT measuring at -200 to $-300 \mathrm{mmHg}$, as well as intravenous antibiotics (cephazolin, $1.0 \mathrm{~g} \mathrm{IV}$, once every $8 \mathrm{~h}$ for 7-10 days), were administered for 7-10 days post-operatively (mean time, 8 days). Close observation was conducted for the early detection of complications (infection, hemorrhage and blistering). During removal of the dressing, an inspection of wound appearance, surface area, depth and exudate amount was conducted. If the granulation formation was favorable with no signs of infection, secondary soft-tissue reconstruction (muscle flap or skin grafting) was performed on the wound deficit.

\section{Results}

Outcomes of treatment. The outcomes of the treatment are summarized in Table II. The mean wound area following resection of the tumor was $73 \mathrm{~cm}^{2}$ (range, $45-110 \mathrm{~cm}^{2}$ ). Upon dressing removal, all cases demonstrated a decreased wound size and depth, favorable granulation formation and a clean skin edge, indicating that the wound bed was feasible for secondary soft-tissue reconstruction. Pain and minor bleeding during dressing removal was recorded in all cases, which was attributable to the growth of granulation into the foam dressing; however, no severe complications were detected. All patients tolerated continuous NPWT well, and no interruption of NPWT occurred due to discomfort. All muscle flaps and skin grafts proved to be viable and healed favorably on discharge. No infection or other major wound complications were recorded. Post-operative CT (6-8 cycles) was administered in 2 patients, and neoadjuvant CT ( 2 cycles) was administered in 1 patient who presented with a recurrent mass in close proximity to major nerves and vessels. Each cycle of treatment consisted of $120 \mathrm{mg} / \mathrm{m}^{2}$ cisplatin, $30 \mathrm{mg} / \mathrm{m}^{2}$ doxorubicin and $2.0 \mathrm{~g} / \mathrm{m}^{2}$ ifosfamide. Post-operative functional and cosmetic outcomes were acceptable, and all patients were satisfied with the outcome of their treatment. During routine follow-up, 1 patient exhibited local recurrence at 12 months after the initial surgery, presenting with recurrent undifferentiated pleomorphic sarcoma at the previous tumor site. Re-excision of the tumor was successfully performed, allowing the limb to be spared. No evidence of remote metastasis was detected, and all patients remained alive at the end of follow-up, which was performed for a mean time of 26 months.

\section{Example cases}

Case 1. A 57-year-old male patient was referred to the Department of Orthopedic Surgery, General Hospital of Jinan 
Table I. Patient data.

\begin{tabular}{lccclcrc}
\hline Case no. & Gender & Age, years & Diagnosis & Tumor site & Skin involvement & Tumor size, $\mathrm{cm}^{3}$ & AJCC stage \\
\hline 1 & Male & 30 & UPS & Right thigh & IU & 461.5 & III \\
2 & Male & 57 & LS & Right knee & Ulcer & 453.5 & III \\
3 & Male & 62 & UPS & Left forearm & IU & Ulcer & IIA \\
4 & Male & 24 & ES & Right leg & Ulcer & III \\
5 & Female & 68 & SS & Right forearm & III & IIB \\
\hline
\end{tabular}

UPS, undifferentiated pleomorphic sarcoma; LS, leiomyosarcoma; ES, epithelioid sarcoma; SS, synovial sarcoma; IU, impending ulceration; AJCC, American Joint Committee on Cancer.

Table II. Treatment and prognosis of patients.

\begin{tabular}{lcccccccc}
\hline $\begin{array}{l}\text { Case } \\
\text { no. }\end{array}$ & Surgery & $\begin{array}{c}\text { Deficit } \\
\text { area, } \mathrm{cm}^{2}\end{array}$ & $\begin{array}{c}\text { Suction } \\
\text { mode }\end{array}$ & $\begin{array}{c}\text { Pressure, } \\
\text { mmHg }\end{array}$ & Surgical margin & CT & $\begin{array}{c}\text { Duration of } \\
\text { NPWT, days }\end{array}$ & Recurrence/metastasis \\
\hline 1 & WR+S+F & 110 & $\mathrm{C}$ & -250 & Negative & DIA & 10 & - \\
2 & WR+S+F & 100 & $\mathrm{C}$ & -300 & Negative & - & 8 & - \\
3 & WR+S & 60 & $\mathrm{C}$ & -200 & Negative & - & 7 & Recurrence \\
4 & WR+S & 48 & $\mathrm{C}$ & -300 & Negative & DIA & 7 & - \\
5 & WR+S & 45 & $\mathrm{C}$ & -300 & Negative & - & 7 & - \\
\hline
\end{tabular}

WR, wide resection; S, split-thickness skin grafting; F, muscle flap; C, continuous mode; DIA, cisplatin + Adriamycin + isofamide; CT, chemotherapy; NPWT, negative pressure wound therapy.
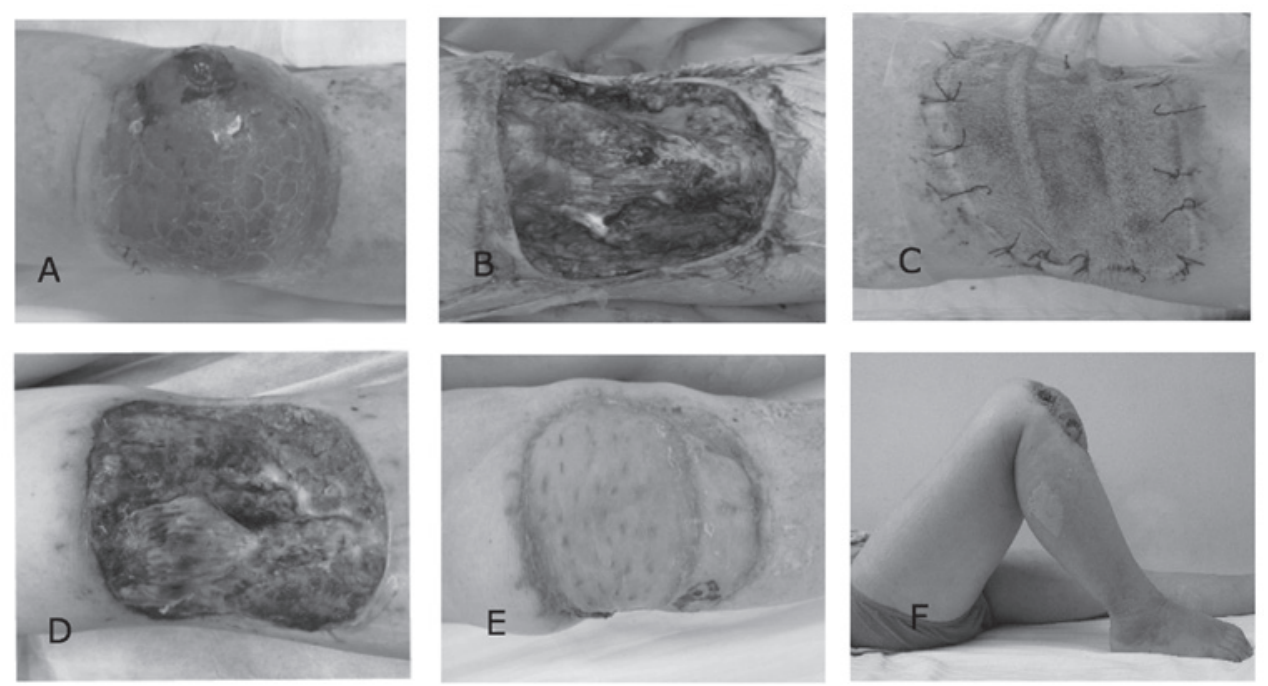

Figure 1. Case 1: Treatment of leiomyosarcoma using negative pressure wound therapy (NPWT). (A) The patient presented with a mass, with ulceration of the right knee. (B) A wound defect with exposed bone surface was caused following excision of the tumor. (C) NPWT was used on the wound bed. (D) The wound bed outcome was favorable following use of NPWT. (E and F) Secondary muscle flap and split-thickness skin grafts were used for wound closure, and the patient outcome was favorable.

Military Commanding Region (Jinan, Shandong) with a mass and ulceration of the right knee (Fig. 1A). The function of the right knee was compromised (range of motion, $5-35^{\circ}$ ), and histological findings revealed spindle cells and myofibrils, indicating a diagnosis of leiomyosarcoma. Pre-operative bacterial culture determined positive results for Staphylococcus epidermidis within the deep tissue of the surgical region. Wide excision of the tumor resulted in a wound defect measuring $100 \mathrm{~cm}^{2}$ and exposed the proximal tibia bone surface (Fig. 1B). To reduce the risk of major wound complications, immediate wound closure was avoided and NPWT was used to cover the wound defect, with simultaneous administration of intravenous antibiotics (Fig. 1C). The microscopic negative surgical margin was determined by pathological examination. Wound 

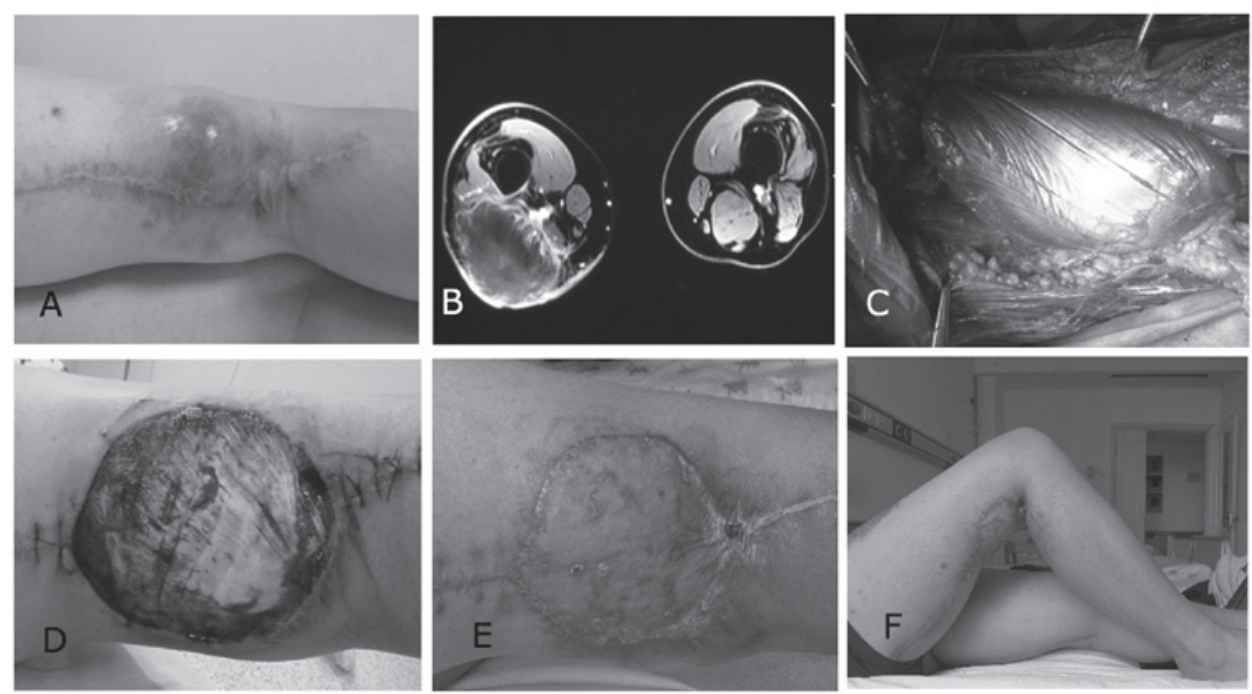

Figure 2. Case 2: A case of recurrent undifferentiated pleomorphic sarcoma treated with negative pressure wound therapy (NPWT). (A) Physical examination indicated a mass with impending ulceration. (B) Magnetic resonance imaging indicated that the tumor was in close proximity to neurovascular structures, therefore, pre-operative chemotherapy was administered to achieve favorable surgical margins. (C) Excision of the tumor resulted in a large defect with exposed nerves and vessels. A gastrocnemius muscle flap was used to cover these structures for subsequent NPWT. (D) Following negative pressure wound therapy, the wound bed was feasible for wound closure with skin grafting. (E anf F) The outcome of treatment was favorable at 12 months post-surgery.

inspection at dressing removal 8 days after surgery indicated that granulation formation was favorable, with no evidence of infection (Fig. 1D). Secondary muscle flap (gastrocnemius muscle) followed by free split-thickness skin grafting was performed. No evidence of recurrence or metastasis was detected. The patient demonstrated improved knee function (range of motion, $0-100^{\circ}$ ), and the appearance of the closed wound defect was acceptable (Fig. 1E and F). The patient was satisfied with the outcome of treatment at 36 months post-surgery.

Case 2. A 30-year-old male patient was diagnosed with recurrent undifferentiated pleomorphic sarcoma and demonstrated impending ulceration following previous surgery on the right distal posterior thigh (Fig. 2A). The function of the patient's right knee was compromised (range of motion, $0-40^{\circ}$ ). Magnetic resonance imaging indicated that the tumor was in close proximity to neurovascular structures, and no tumor encasement of major nerves and vessels was detected (Fig. 2B). To achieve favorable surgical margins, neoadjuvant $\mathrm{CT}$ was commenced $\left(120 \mathrm{mg} / \mathrm{m}^{2}\right.$ cisplatin, $30 \mathrm{mg} / \mathrm{m}^{2}$ doxorubicin and $2.0 \mathrm{~g} / \mathrm{m}^{2}$ ifosfamide, for 2 cycles pre-operatively at an interval of 7 days). Aggressive excision of the tumor resulted in a wound defect measuring $110 \mathrm{~cm}^{2}$, with exposed neurovascular structures. A gastrocnemius muscle flap was immediately used to separate the neurovascular structures from the subsequent negative pressure (Fig. 2C). NPWT was used to cover the wound defect as preparation for secondary wound closure. Microscopic negative surgical margins were determined by pathological examination. Wound inspection at dressing removal 10 days after surgery indicated that the wound bed was feasible for skin grafts, with favorable granulation formation (Fig. 2D). Following split-thickness skin grafting, the patient was administered 6 cycles of post-operative CT $\left(120 \mathrm{mg} / \mathrm{m}^{2}\right.$ cisplatin, $30 \mathrm{mg} / \mathrm{m}^{2}$ doxorubicin and $2.0 \mathrm{~g} / \mathrm{m}^{2}$ ifosfamide). The cosmetic and functional outcomes (range of motion, -5 to $90^{\circ}$ ) were acceptable (Fig. $2 \mathrm{E}$ and F). The patient returned to normal daily activities and was satisfied with the outcome of treatment at 12 months post-surgery.

\section{Discussion}

The surgical approach to the treatment of STS of the extremities has undergone significant change over the past decade. Aggressive resection of the tumor along with the pseudocapsule may assist in achieving microscopic negative surgical margins, which is deemed to be the strongest predictor of patient prognosis (14). With advances in multidisciplinary personalized treatment of STS, limb-sparing surgeries have become the preferred choice of treatment over amputations (5). However, extensive tissue defects following surgery have challenged the efficacy and feasibility of limb salvage, as under certain circumstances primary closure is infeasible due to insufficient soft tissue and exposed bone, tendons, nerves or vessels (15). Conventional measures to treat such wound defects following the wide excision of tumors are primarily composed of a variety of soft-tissue reconstruction methods. Previous studies have reported the use of various flaps (local, regional, distant and free) and skin grafts (split-thickness and full-thickness) as an important adjunct to treatment for STS $(16,17)$. However, the aforementioned complex techniques may predispose patients to an increased risk of major wound complications, including infection, seromas, hematomas and necrotic flaps. A number of studies have reported that the complication rates associated with reconstruction may be as high as $16-56 \%(18,19)$. In the present study, all STS patients presented with ulceration or impending ulceration. This increased the risk of post-operative infection due to potential bacterial colonization or inflammation within the surgical field. These major wound complications may result in delayed post-operative adjuvant therapy and an increased risk of amputation, and may have a detrimental impact on oncological outcomes. 
In the present study, in order to manage wound defects while preventing the occurrence of major wound complications, NPWT was applied to facilitate wound closure. NPWT was used as a bridge for wound closure in STS affecting the skin, as the cases in the present study were expected to demonstrate bacterial contamination or inflammation within the surgical field. Such patients are exposed to a considerable risk of post-operative infection; therefore, proactive wound care measures reducing the risk of infection are imperative. The present study used NPWT as a reliable method of assisting in reducing the risk of infection, as it is able to separate the wound from contamination and has been observed to be efficient in controlling bacterial proliferation (20). Li et al (20) investigated the efficacy of regulated NPWT in the treatment of infected blast injury in swine. The results of this study indicated that NPWT reduced the bacterial load efficiently compared with gauze dressing treatments (20). Blum et al (21) compared the efficacy of NPWT and conventional dressings for the treatment of open tibial fractures that underwent delayed soft-tissue coverage, and NPWT was observed to reduce the risk of deep infection by almost $80 \%$ (21). As well as preventing infection and decreasing the bacterial load, NPWT has demonstrated a wide variety of merits in the treatment of complex wound defects. Previous studies have reported that NPWT is able to decrease wound size, maintain a moist atmosphere for wound healing, reduce edema and assist with the closure of areas not fully closed in the wound, also termed dead cavities $(8,22)$. Notably, microscopically NPWT is able to promote granulation, vascularization, epithelialization and the synthesis of fibrin within the wound $(23,24)$. However, there is little relevant literature regarding the use of NPWT as an adjunct to wound closure in patients with tumors. Oh et al (25) assessed the efficacy of NPWT in conjunction with secondary full-thickness skin grafting for the treatment of melanoma on the foot, and observed acceptable functional and cosmetic outcomes. Katz et al (26) used NPWT as an adjunct to surgery for lymphangioma in children. The results of this study indicated that NPWT was effective and safe for post-operative wound closure, and that NPWT also decreased the risk of recurrence and infection (26). NPWT means that daily dressing changes are unnecessary, as accumulation of fluid at wound sites is deterred by the drainage; thus, the labor required from clinicians is reduced (22). Therefore, the aforementioned characteristics make NPWT an ideal adjunct to the management of wound defects following excision of STS of the extremities with potential risk of major wound complications.

Treatment of extremity STSs consists of a meticulous pre-operative evaluation, complete removal of the tumor and surrounding healthy tissue, soft-tissue reconstruction and adjuvant therapies, including RT or CT $(2,3)$. As STSs are composed of a heterogeneous group of disease subtypes with various manifestations, a pre-operative evaluation is of importance and determines the treatment strategy. Evaluation must assess tumor involvement with peripheral major neurovascular structures and whether the tumor arises from or is encasing the major nerves or blood vessels; if the expected function of the distal limb is predicted to be poor, amputation may be preferable to limb-sparing surgery (27). Previously, a number of attempts have been made to reconstruct the nerves and vessels, so as to expand the indications for limb-sparing surgery among patients with STSs of the extremities. In cases with tumor involvement of the vessels, reversed saphenous vein grafts, femoral venous grafts or synthetic grafts have been used as options for limb salvage surgery (28). However, post-operative functional results are less predictable, and the patients exhibit high risks of complications and amputation (28). Nerve transfer may be a promising alternative for amputation in cases with nerve encasement; however, this is currently a complex technique, which is only used for brachial plexus reconstruction (29). The feasibility of nerve transfer in treating defects following a wide resection of an STS remains to be demonstrated. Notably, all choices regarding limb-sparing surgery should not be made until the goal of negative surgical margins is achievable. In the present study, a patient with recurrent undifferentiated pleomorphic sarcoma in close proximity to major nerves and vessels of the lower extremities was administered neoadjuvant CT to achieve favorable surgical margins. This procedure proved to be effective during surgery, and the goal of a wide excision of the tumor was achieved.

When managing large wound defects with or without exposed bone, tendons, nerves and vessels following resection of the tumor, soft-tissue reconstruction should be conducted. In clinical practice, cases with indications for reconstruction are not rare in the treatment of STS of the extremities, and a previous study reported that 40/100 consecutive patients with STS underwent immediate soft-tissue reconstruction (18). The spectrum of associated surgical techniques involves flaps (local, regional, distant and free flaps) and split-thickness or full-thickness skin grafts (6). The specific techniques selected are dependent on a comprehensive evaluation of the defect size and depth, the location of tumor, the tumor proximity to neurovascular structures and the overall health status (30). In the current study, NPWT allowed for secondary soft-tissue reconstruction for cases with a high risk of post-operative wound complications. Furthermore, using a muscle flap as coverage on the exposed neurovascular structures allowed for application of NPWT on the wound defect, as exposed nerves and vessels are vulnerable to negative pressure, and are considered to be a contraindication for NPWT (22).

A number of factors should be taken into account when implementing NPWT on wound defects following wide tumor resection. The goal of using NPWT in the current study was to prepare the wound bed for secondary soft-tissue reconstruction, while reducing the risk of infection and other major wound complications. However, the optimal suction protocol to be applied to this situation remains to be elucidated, and the reported methods are primarily empirical. Previous studies regarding the use of NPWT primarily concern traumatic wounds with or without infection, and the most frequently used pressure is -80 to $-125 \mathrm{mmHg}$ (31). In cases with fragile wound edges, low perfusion, poor tolerability or skin grafts, a minor pressure $(<125 \mathrm{mmHg})$ is often attempted (8). Furthermore, negative pressure protocols are typically used at one of two settings, namely, continuous or intermittent. In one previous study, intermittent suction was observed to cause a more profound tissue response compared with conventional methods; however, this suction mode may cause discomfort and intolerability in patients (32). In the present study, continuous negative pressure at a relatively high level of -200 to $300 \mathrm{mmHg}$ was used on the wound beds. This suction protocol was used as it had previously 
been observed to be effective in clinical practice and as stronger suction was not considered since intense negative pressure may increase the risk of hemorrhage (10). Intermittent suction was additionally avoided due to potential patient intolerability. The duration of NPWT is dependent on the goal of treatment and the wound status. In the present study, devices were removed on post-operative days 7-10, when granulation formation was favorable. According to observations made at the point of foam dressing removal, the drainage protocol used in the present study was effective in reducing edema, removing exudation, promoting granulation formation and assisting with the prevention of wound infection. An increased duration of NPWT was avoided, as the risk of infection increases along with prolonged drainage duration, and early initiation of post-operative adjuvant therapy additionally required a shortened drainage duration (18).

Potential complications associated with NPWT should be taken into account during clinical practice. The associated complications include hemorrhage, blistering, odors, skin erosion around the suction tube, sepsis and obstruction of the suction tube (33). A number of the aforementioned complications, although rarely observed, may be severe; thus, close observation and assessment of the wound throughout the entire process of NPWT is necessary. In the present study, frequent observation of exudation, wound edges and the patency of the drainage tube was conducted. No major complications were detected, whereas pain and minor bleeding during dressing removal were recorded in all cases; this was in accordance with the majority of the outcomes in previous studies $(11,22)$. Pain and minor bleeding were attributed to the growth of granulation tissue into the foam dressing; however, all patients responded to these complications with good tolerability.

Notably, the sample size in the current study was small, as extremity STSs with skin involvement account for a small fraction of the total STS patients admitted by the Department of Orthopedic Surgery, General Hospital of Jinan Military Commanding Region. In the present study, when NPWT was used as an adjunct to the treatment of extremity STS with ulceration or impending ulceration, the outcomes of treatment were favorable. However, studies on larger sample sizes and with a control group are required to test the efficacy of NPWT in decreasing major wound complications compared with conventional treatment.

In conclusion, when wide tumor excision and negative surgical margins are achievable, NPWT in conjunction with soft-tissue reconstruction may be a reliable and safe adjunct to the management of large soft-tissue deficits caused by aggressive excision of extremity STS with ulceration or impending ulceration.

\section{References}

1. Rosenberg AE: WHO classification of soft tissue and bone, fourth edition: Summary and commentary. Curr Opin Oncol 25: $571-573,2013$

2. Kneisl JS, Coleman MM and Raut CP: Outcomes in the management of adult soft tissue sarcomas. J Surg Oncol 110: 527-538, 2014.

3. Gronchi A, Colombo C and Raut CP: Surgical management of localized soft tissue tumors. Cancer 120: 2638-2648, 2014.

4. Trovik CS, Bauer HC, Alvegård TA, Anderson H, Blomqvist C, Berlin O, Gustafson P, Saeter G and Wallöe A: Surgical margins, local recurrence and metastasis in soft tissue sarcomas: 559 surgically-treated patients from the Scandinavian Sarcoma Group Register. Eur J Cancer 36: 710-716, 2000.
5. Williard WC, Collin C, Casper ES, Hajdu SI and Brennan MF: The changing role of amputation for soft tissue sarcoma of the extremity in adults. Surg Gynecol Obstet 175: 389-396, 1992.

6. Talbot SG, Athanasian EA, Cordeiro PG and Mehrara BJ: Soft tissue reconstruction following tumor resection in the hand. Hand Clin 20: vi, 181-202, 2004.

7. Baldini EH, Lapidus MR, Wang Q, Manola J, Orgill DP, Pomahac B, Marcus KJ, Bertagnolli MM, Devlin PM, George S, et al: Predictors for major wound complications following preoperative radiotherapy and surgery for soft-tissue sarcoma of the extremities and trunk: Importance of tumor proximity to skin surface. Ann Surg Oncol 20: 1494-1499, 2013.

8. Jeong HS, Lee BH, Lee HK, Kim HS, Moon MS and Suh IS: Negative pressure wound therapy of chronically infected wounds using 1\% acetic Acid irrigation. Arch Plast Surg 42: 59-67, 2015.

9. Orgill DP, Manders EK, Sumpio BE, Lee RC, Attinger CE, Gurtner GC and Ehrlich HP: The mechanisms of action of vacuum assisted closure: More to learn. Surgery 146: 40-51, 2009.

10. Karaaslan F, Erdem Ş and Mermerkaya MU: Wound management with vacuum-assisted closure in postoperative infections after surgery for spinal stenosis. Int Med Case Rep J 8: 7-11, 2014.

11. WuCC,ChewKY,ChenCCandKuoYR:Antimicrobial-impregnated dressing combined with negative-pressure wound therapy increases split-thickness skin graft engraftment: A simple effective technique. Adv Skin Wound Care 28: 21-27, 2015.

12. Putnis S, Khan WS and Wong JM: Negative pressure wound therapy - a review of its uses in orthopaedic trauma. Open Orthop J 8: 142-147, 2014

13. Lahat G, Tuvin D, Wei C, Anaya DA, Bekele BN, Lazar AJ, Pisters PW, Lev D and Pollock RE: New perspectives for staging and prognosis in soft tissue sarcoma. Ann Surg Oncol 15: 2739-2748, 2008.

14. Gronchi A, Verderio P, De Paoli A, Ferraro A, Tendero O, Majò J, Martin J, Comandone A, Grignani G, Pizzamiglio S, et al: Quality of surgery and neoadjuvant combined therapy in the ISG-GEIS trial on soft tissue sarcomas of limbs and trunk wall. Ann Oncol 24: 817-823, 2013.

15. Barwick WJ, Goldberg JA, Scully SP and Harrelson JM: Vascularized tissue transfer for closure of irradiated wounds after soft tissue sarcoma resection. Ann Surg 216: 591-595, 1992.

16. Boyce DE and Shokrollahi K: Reconstructive surgery. BMJ 332: 710-712, 2006.

17. Turner AJ and Parkhouse N: Revisiting the reconstructive ladder. Plast Reconstr Surg 118: 267-268, 2006.

18. Lohman RF, Nabawi AS, Reece GP, Pollock RE and Evans GR: Soft tissue sarcoma of the upper extremity: A 5-year experience at two institutions emphasizing the role of soft tissue flap reconstruction. Cancer 94: 2256-2264, 2002.

19. Geller DS, Hornicek FJ, Mankin HJ and Raskin KA: Soft tissue sarcoma resection volume associated with wound-healing complications. Clin Orthop Relat Res 459: 182-185, 2007.

20. Li J, Topaz M, Tan H, Li Y, Li W, Xun W, Yuan Y, Chen S and Li X: Treatment of infected soft tissue blast injury in swine by regulated negative pressure wound therapy. Ann Surg 257: 335-344, 2013.

21. Blum ML, Esser M, Richardson M, Paul E and Rosenfeldt FL: Negative pressure wound therapy reduces deep infection rate in open tibial fractures. J Orthop Trauma 26: 499-505, 2012.

22. de Laat EH, van den Boogaard MH, Spauwen PH, van Kuppevelt DH, van Goor H and Schoonhoven L: Faster wound healing with topical negative pressure therapy in difficult-to-heal wounds: A prospective randomized controlled trial. Ann Plast Surg 67: 626-631, 2011.

23. Kim BS, Choi WJ, Baek MK, Kim YS and Lee JW: Limb salvage in severe diabetic foot infection. Foot Ankle Int 32: 31-37, 2011.

24. Scherer SS, Pietramaggiori G, Mathews JC, Prsa MJ, Huang S and Orgill DP: The mechanism of action of the vacuum-assisted closure device. Plast Reconstr Surg 122: 786-797, 2008.

25. Oh BH, Lee SH, Nam KA, Lee HB and Chung KY: Comparison of negative pressure wound therapy and secondary intention healing after excision of acral lentiginous melanoma on the foot. Br J Dermatol 168: 333-338, 2013.

26. Katz MS, Finck CM, Schwartz MZ, Moront ML, Prasad R, Timmapuri SJ and Arthur LG: Vacuum-assisted closure in the treatment of extensive lymphangiomas in children. J Pediatr Surg 47: 367-370, 2012.

27. Ferguson PC, Kulidjian AA, Jones KB, Deheshi BM and Wunder JS: Peripheral nerve considerations in the management of extremity soft tissue sarcomas. Recent Results Cancer Res 179: 243-256, 2009. 
28. Mahendra A, Gortzak Y, Ferguson PC, Deheshi BM, Lindsay TF and Wunder JS: Management of vascular involvement in extremity soft tissue sarcoma. Recent Results Cancer Res 179: 285-299, 2009.

29. Lee SK and Wolfe SW: Nerve transfers for the upper extremity: New horizons in nerve reconstruction. J Am Acad Orthop Surg 20: 506-517, 2012.

30. Wong JC and Abraham JA: Upper extremity considerations for oncologic surgery. Orthop Clin North Am 45: 541-564, 2014.

31. Borgquist $\mathrm{O}$, Ingemansson $\mathrm{R}$ and Malmsjö $\mathrm{M}$ : Individualizing the use of negative pressure wound therapy for optimal wound healing: A focused review of the literature. Ostomy Wound Manage 57: 44-54, 2011.
32. Younan G, Ogawa R, Ramirez M, Helm D, Dastouri P and Orgill DP: Analysis of nerve and neuropeptide patterns in vacuum-assisted closure-treated diabetic murine wounds. Plast Reconstr Surg 126: 87-96, 2010.

33. Huang C, Leavitt T, Bayer LR and Orgill DP: Effect of negative pressure wound therapy on wound healing. Curr Probl Surg 51: 301-331, 2014. 